\title{
A group-enhanced sprint interval training program for amateur athletes
}

\author{
Luc J. Martin, Scott H. Anderson, Matthew S. Schmale, Jillian R. Hallworth, and Tom J. Hazell
}

\begin{abstract}
Sprint interval training (SIT) can elicit improvements in aerobic and anaerobic capacity. While variations in SIT protocols have been investigated, the influence of social processes cannot be overlooked. As research supports the use of groups to influence individual cognitions and behaviours, the current project assessed the effectiveness of a group-based intervention with participants conducting SIT. Specifically, 53 amateur athletes (age, $21.9 \pm 2.9$ years; 53\% females) took part in a 4-week training program (3 sessions per week, 30-s "all-out" efforts with 4 min active recovery, repeated 4-6 times per session), and were assigned to "true group", aggregate, or individual conditions. Results indicated no significant differences between groups for the physiological measures. With regards to training improvements from baseline for all participants — regardless of condition significant main effects for time were identified for maximal oxygen uptake $\left(2.5-2.8 \mathrm{~mL} \cdot \mathrm{kg}^{-1} \cdot \mathrm{min}^{-1}, p<0.001, \eta^{2}=0.03\right)$, time-trial performance (14-32 s, $p<0.001, \eta^{2}=0.37$ ), and anaerobic power $\left(1.1-1.7 \mathrm{k} \cdot \mathrm{h}^{-1}, p<0.001, \eta^{2}=0.66\right)$. With regards to the psychological measures, significant main effects between groups were found for motivation $\left(p=0.033, \eta^{2}=0.13\right)$, task selfefficacy $\left(p=0.018, \eta^{2}=0.15\right)$, and scheduling self-efficacy $\left(p=0.003, \eta^{2}=0.22\right)$. The true group experienced greater improvements in motivation than the individual condition, but the aggregate and individual conditions demonstrated greater increases in task and scheduling self-efficacy. Though the SIT paradigm employed induced training improvements similar to previous work, the group intervention was not able to further these improvements.
\end{abstract}

Key words: group dynamics, high-intensity interval training, team building, performance.

Résumé : L’entraînement par intervalle au sprint (« SIT ») peut susciter des améliorations des capacités aérobie et anaérobie. La documentation rapporte divers protocoles SIT; toutefois, on ne peut pas négliger l'influence des processus sociaux. D'après des études, l'utilisation de groupes produit un effet sur les cognitions et les comportements des individus; cette étude évalue donc l'efficacité d'une intervention en groupe chez des participants à un SIT. Dès lors, 53 athlètes amateurs (âge 21,9 $\pm 2,9$ ans; 53 \% de femmes) participent à 4 semaines d'entraînement ( 3 séances par semaine comprenant des exercices de $30 \mathrm{~s}$ à fond de train et 4 min de récupération active à raison de 4-6 répétitions par séance) et sont répartis dans trois groupes : "réel », regroupement et d'individus. D'après les résultats, il n'y a pas de différences significatives entre les groupes sur le plan des mesures physiologiques. À propos des améliorations de tous les participants depuis le début, indépendamment de la condition, on enregistre un effet significatif du temps sur le consommation maximale d'oxygène $\left(2,5-2,8 \mathrm{~mL} \cdot \mathrm{kg}^{-1} \cdot \mathrm{min}^{-1}, p<0,001, \eta^{2}=0,03\right)$, sur la performance au contre-la-montre (14-32 s, $\left.p<0,001, \eta^{2}=0,37\right)$ et sur la puissance anaérobie $\left(1,1-1,7 \mathrm{k} \cdot \mathrm{h}^{-1}, p<0,001, \eta^{2}=0,66\right)$. En ce qui concerne les mesures psychologiques, on enregistre entre les groupes un effet significatif de la motivation $\left(p=0,033, \eta^{2}=\right.$ $0,13)$, l'autoefficacité de la tâche $\left(p=0,018, \eta^{2}=0,15\right)$ et l'autoefficacité de la planification $\left(p=0,003, \eta^{2}=0,22\right)$. Le groupe " réel » affiche une plus grande amélioration de la motivation que le groupe d'individus; dans les conditions de regroupement et d'individualité, on observe une plus grande amélioration de l'autoefficacité de la tâche et de la planification. Même si le paradigme de SIT utilisé dans cette étude suscite des améliorations par l'entraînement similaires aux résultats d'études antérieures, l'intervention en groupe ne pousse pas plus loin les améliorations. [Traduit par la Rédaction]

Mots-clés : dynamique de groupe, entraînement par intervalle d’intensité élevée, travail d'équipe, performance.

\section{Introduction}

High-intensity interval training (HIIT) paradigms involve short exercise bouts between $80 \%-100 \%$ maximal oxygen uptake $\left(\dot{V}_{2 \max }\right)$ or maximum heart rate (HR) interspersed with active rest of lowintensity activity (Weston et al. 2014a). Sprint interval training (SIT) is an intense form of HIT comprising repeated maximal "all-out" exercise efforts followed by short periods of active recovery, such as walking (Gibala et al. 2006, 2014; Hazell et al. 2010; Weston et al. 2014a). Recent research involving SIT protocols has demonstrated improvements in cardiorespiratory fitness, cardiovascular health, glycemic control, and body composition (Gibala et al. 2014), which are comparable or superior to those obtained through more traditional forms of continuous exercise (Burgomaster et al. 2008; Gibala et al. 2006, MacPherson et al. 2011). However, because of its intensity - and by extension, tolerability and adherence issues the utility of SIT has been questioned (Hardcastle et al. 2014). Interestingly, others suggest it is too early to make such claims, and that further research is warranted (Del Vecchio et al. 2015; Jung et al. 2015). In recognizing these varying perspectives, researchers are tasked with developing protocols to maintain such training benefits, but with a specific focus on enriching the exercise experience.

Received 3 December 2015. Accepted 22 March 2016.

L.J. Martin. School of Kinesiology and Health Studies, Queen's University, Kingston, ON K7L 3N6, Canada.

S.H. Anderson, M.S. Schmale, and J.R. Hallworth. Department of Kinesiology and Physical Education, University of Lethbridge, Lethbridge,

AB T1K 3M4, Canada.

T.J. Hazell. Department of Kinesiology and Physical Education, Wilfrid Laurier University, Waterloo, ON N2L 3C5, Canada.

Corresponding author: Luc J. Martin (email: luc.martin@queensu.ca).

Copyright remains with the author(s) or their institution(s). Permission for reuse (free in most cases) can be obtained from RightsLink. 


\section{Pagination not final (cite DOI) / Pagination provisoire (citer le DOI)}

There are numerous opportunities for variations in SIT protocols, such as altering the length of sprints and recovery durations (Gillen et al. 2014; Hazell et al. 2010; Zelt et al. 2014); however, the influence of social processes that occur during training cannot be overlooked. In fact, the need for group membership has been highlighted as a prominent human characteristic (Forsyth and Burnett 2010), and its salience has recently been supported: "the primary rationale for using small group interventions is that the group can exert a positive influence on individual behaviour" (Estabrooks et al. 2014). Indeed, the benefits obtained from physical activity interventions delivered to groups have been identified (Dishman and Buckworth 1996). Interestingly, despite the support for group-based protocols across numerous populations ranging from community to clinical based (Estabrooks et al. 2006, 2008, 2011, 2014; Estabrooks and Glasgow 2006; Estabrooks and Smith-Ray 2005), they were nonetheless conducted in an exercise context. While there is less support with athlete populations, similar trends do exist. As several examples, the closeness or unity perceived by team members is positively related to individual efficacy beliefs (Marcos et al. 2010), work output (Prapavessis and Carron 1997), and intentions to continue involvement (Spink 1995; Viglietta et al. 2012).

When inferring the influence of social processes, however, we must cogitate the presence of all others, and not simply those considered to be members of a similar group. The concept of a "true group" pertains to individuals who have a common identity, established group goals, and who perceive themselves to be a group (Burke et al. 2006). Conversely, aggregates of people are individuals who are in the exercisers' environment, but have no connection to one another (Lox et al. 2014). While the presence of these random co-exercisers can increase levels of effort and motivation (Martin Ginis and Mack 2012; Rhea et al. 2003), interventions targeting connections and support (i.e., true groups), likely elicit improvements superior to those derived from aggregates of people (Burke et al. 2006; Estabrooks et al. 2014).

An enduring characteristic that contributes to the forces enacted on individuals based on group membership is that they perceive themselves to be a group, or more specifically, they experience entitativity or "groupness" (Campbell 1958; Spink et al. 2010). Conceptually, whereas a true group represents the presence of certain variables, such as the demonstration of common fate, mutual benefit, social structure, group processes, and self-categorization (Carron and Eys 2012), groupness represents the degree to which they are present. This degree is important in the activity setting, as increased levels of groupness have been found to contribute to important individual cognitions and behaviours (Crozier et al. 2012; Priebe et al. 2011; Spink et al. 2010).

Therefore, the current project sought to exploit the social environment to improve training through a group-enhanced SIT program for amateur athletes. Our objective was to determine whether we could improve both physiological $\left(\mathrm{VO}_{2 \max }\right.$, aerobic, and anaerobic performance) and psychological (motivation and efficacy beliefs) outcomes for those involved. It was hypothesized that participants assigned to the true group condition would demonstrate greater improvements in comparison with those training with co-exercisers (i.e., aggregates) and alone (i.e., individual).

\section{Materials and methods}

Fifty-three amateur athletes (28 females; age, $21.9 \pm 2.9$ years; height, $174.5 \pm 26.1 \mathrm{~cm}$; body mass, $72.4 \pm 11.8 \mathrm{~kg}$; mean \pm SD) from Canadian Interuniversity Sport (CIS), the Canadian Collegiate Athlete Association (CCAA), and competitive adult club programs volunteered to participate in this study. These amateur athletes were involved in a variety of sports (e.g., soccer, basketball, hockey, volleyball, swimming, boxing, skiing, and track and field) and were eligible to participate if they met 2 conditions: they were healthy individuals (assessed by the Physical Activity Readiness health questionnaire) and were not involved in other systematic exercise training over the duration of the study. Dietary and physical activity patterns were maintained throughout the study, with no alcohol or caffeine permitted for $24 \mathrm{~h}$, no physical exercise for $36 \mathrm{~h}$, and no heavy meals for $2 \mathrm{~h}$ before each training or testing session.

\section{Recruitment}

Once approval was granted from the research ethics board at the host institution, participant recruitment commenced. CIS and CCAA coaches as well as representatives from local competitive sport organizations were contacted to gauge their interest in allowing the research group to approach their athletes. Once approval was obtained, athletes were told of the purpose of the project and asked to contact the research group if they were interested in participating. Interested volunteers were then briefed on the protocol, screened for eligibility, and asked to provide informed consent.

\section{Familiarization session}

Prior to baseline testing, participants were introduced to testing and experimental procedures to minimize learning effect. During this session, participants were given directions on how to safely use the curved, self-propelled treadmill. Each participant was allotted as much time as desired to become familiar with the treadmill and was directed to practice accelerations. Participants were fitted and acquainted with the facemasks used for $\dot{V} \mathrm{O}_{2 \max }$ testing and those who had never used a traditional motorized treadmill (used for $\dot{V O}_{2 \max }$ assessments) were given time to become comfortable with running on the device. Pacing strategies for the $2000-\mathrm{m}$ time trial were also explained (Willoughby et al. 2016).

\section{Pre- and post-testing}

Testing procedures were identical before and after training, with a minimum of $48 \mathrm{~h}$ separating testing and training sessions. Testing was performed over 2 days (with a minimum of $48 \mathrm{~h}$ between sessions), and was carried out in the same order for all participants and consisted of time-trial performance on 1 day, and $\mathrm{VO}_{2 \max }$ and anaerobic running performance on a different day. Participants were given $20 \mathrm{~min}$ to recover between the $\dot{V}_{2 \max }$ and anaerobic running assessments based on pilot testing and data demonstrating $20 \mathrm{~min}$ to be sufficient to fully resynthesize intramuscular phosphocreatine levels following an exhaustive bout of exercise (Harris et al. 1976).

\section{Manipulation check}

To demonstrate that the group-based intervention was effective, athletes from the true group and aggregate conditions completed questionnaires that assessed perceptions of groupness at baseline and post-training. Note that athletes assigned to the individual condition were not asked to complete these questionnaires as they trained in isolation. In order for the intervention to facilitate physiological and psychological improvements, it was hypothesized that group members should perceive themselves as a true group, and by extension, demonstrate greater levels of groupness.

\section{Measures}

\section{Physiological measures}

\section{Anthropometry}

Participants had their height (nearest $0.1 \mathrm{~cm}$ ) and body mass (nearest $0.1 \mathrm{~kg}$ ) measured using a mechanical beam scale (Healtho-meter Professional, Sunbeam Products Inc., Ill., USA).

\section{Running $\dot{\mathrm{V}} \mathrm{O}_{2 \max }$ test}

Participants performed a warm-up run (5 min) on the treadmill (TMX22, Trackmaster, Jas Fitness Systems, Kans., USA) at $8 \mathrm{~km} \cdot \mathrm{h}^{-1}$ (women) or $9.7 \mathrm{~km} \cdot \mathrm{h}^{-1}$ (men). Following the warm-up, participants performed a continuous and incremental speed treadmill test to determine $\dot{V} \mathrm{O}_{2 \max }$. The protocol began at $8.9 \mathrm{~km} \cdot \mathrm{h}^{-1}$ (women) or $10.5 \mathrm{~km} \cdot \mathrm{h}^{-1}$ (men) and the speed increased by $0.8 \mathrm{~km} \cdot \mathrm{h}^{-1}$ every minute until volitional exhaustion. Oxygen consumption $\left(\mathrm{V}_{2}\right)$ was col- 


\section{Pagination not final (cite DOI) / Pagination provisoire (citer le DOI)}

lected continuously and analyzed using an online breath-by-breath gas collection system (Quark CPET, Cosmed, Calif., USA). HR was recorded throughout the test using an integrated HR monitor. $\dot{V} \mathrm{Omax}_{\text {max }}$ (greatest 30-s average) was established by the presence of a plateau in the $\dot{V} \mathrm{O}_{2}$ values $\left(<1.35 \mathrm{~mL} \cdot \mathrm{kg}^{-1} \dot{\mathrm{VO}} \mathrm{O}_{2}\right.$ increase) despite an increase in speed or when 2 of the following criteria were obtained: a respiratory exchange ratio value $\geq 1.10$, the achievement of a maximal HR within 10 beats. $\mathrm{min}^{-1}$ of the predicted maximal HR (220 age), and/or visible subject exhaustion (Midgley et al. 2007).

\section{Running time-trial (2000 m)}

Participants were instructed to complete a $2000-\mathrm{m}$ self-paced time-trial on a $200-\mathrm{m}$ indoor track as quickly as possible with no feedback. Exercise time was recorded upon the completion of the test to the nearest $0.1 \mathrm{~s}$.

\section{Anaerobic running test}

A 30-s all-out sprint on a self-propelled treadmill (Curve, Woodway, Wis., USA) was performed, where the participants serve as the power source - the treadmill moves as fast as the athlete can run (Willoughby et al. 2016). Participants were instructed to run as fast as they could for the entire 30-s sprint and verbal encouragement was provided throughout. Peak speed $\left(\mathrm{km} \cdot \mathrm{h}^{-1}\right)$ was recorded as the fastest speed attained in the first 5-10 s.

\section{Psychological measures}

\section{Intrinsic motivation}

Motivation was assessed using the 4-item subscale of intrinsic motivation from the Behavioral Regulation in Exercise Questionnaire (BREQ-2). The items are measured on a 5-point Likert type scale anchored at the extremes with zero (not true for me) to 4 (very true for me), where higher scores reflect greater intrinsic motivation. Previous research has supported the factor structure and validity of the BREQ-2 with similar populations (Markland and Tobin 2004), and adequate reliability was demonstrated with the current sample $(\alpha>0.70)$.

\section{Task and scheduling self-efficacy}

Task and scheduling self-efficacy were assessed by a 10-item $100 \%$ confidence scale anchored at the extremes with $0 \%$ (no confidence) and $100 \%$ (complete confidence) based on recommendations from previous research (Rodgers et al. 2002; Rodgers and Sullivan 2001). All items had a consistent stem (i.e., "How confident are you that you can") with the remainder of the item being tailored to task ( $n=5$; e.g., "complete the sprint interval workout?") or scheduling ( $n=5$; e.g., "exercise 3 times per week for the next 4 weeks?") beliefs. With the current sample, the reliability for both subscales was adequate $(\alpha>0.70)$.

\section{Groupness}

Groupness was used as the manipulation check between the true group and aggregate conditions. It was assessed using a 5-item measure (Spink et al. 2010), with each item representing 1 of the variables previously described as reflecting a level of groupness (i.e., common fate, social structure, mutual benefit, group processes, and self-categorization (Carron and Hausenblas 1998). Each item is based on a 9-point Likert type scale anchored with 1 (not at all) and 9 (very much so), thus, higher scores reflect greater perceptions of groupness. The scale demonstrated adequate reliability with the current sample $(\alpha>0.70)$.

\section{Training program}

After baseline procedures were complete, participants were assigned to 1 of 3 training groups: (i) true group $(n=20)$, (ii) aggregate $(n=18)$, and (iii) individual $(n=15)$. Participants were ranked based on $\dot{V} \mathrm{O}_{2 \max }$, sex, and time-trial performance and then with stratified into groups. Training commenced for all groups $\sim 48 \mathrm{~h}$ after the last baseline test and consisted of 3 training sessions per week
( $\sim 1.5 \mathrm{~h})$ over a 4 -week period ( $\sim 6 \mathrm{~h})$, with $48-72 \mathrm{~h}$ of recovery between training sessions. All training bouts were conducted on a selfpropelled treadmill, with 4-6 sets of 30-s all-out efforts separated by $4 \mathrm{~min}$ of active recovery (i.e., light walking). With regard to the training, all participants completed the 12 bouts, and they increased in intensity with 4 sprints per bout in week 1,5 sprints per bout in week 2, and 6 sprints per bout in weeks 3 and 4 .

\section{Group-based intervention}

\section{True group condition}

In addition to the training protocols, those in the true group condition received a group-based intervention grounded in an established framework (Carron and Spink 1993). Specifically, group sessions $(\sim 1 \mathrm{~h})$ were held with each true group $(n=5)$ prior to the beginning of the training program. This meeting allowed members to introduce themselves and discuss their motivations for taking part in the training. Once this had occurred, 4 aspects from the framework involving inputs, throughputs, and outputs were targeted (Carron and Spink 1993). With regard to inputs, the focus was on the group's environment and structure. For the environment, group members collectively established a team name and motto, which were subsequently printed on the training apparel (t-shirt) that was provided to them. As for group structure, each athlete described their ideal workout environment, which was used to establish expectations and norms for training (e.g., attendance, arrival time, attitudes, motivational strategies). In terms of throughputs, the focus was on processes such as group goals and support. Group goals were established by providing individual members with their baseline physiological measures (athletes in all conditions were provided with their scores), whereby a short discussion ensued, and athletes were asked to establish difficult but attainable objectives. Once these were set, the group collectively agreed on group-level objectives (which would be a summation of their individual final assessments), and only those were posted on a training board. To emphasize support and facilitate communication, members were asked to distribute contact information (e.g., email address, phone number). In addition, a rotating "buddy system" meant that each group member would be training directly with another member in terms of timing their sessions and recording their speeds (a research assistant recorded the peak speed for research purposes). Based on the theorizing from Carron and Spink (1993), the implementation of these inputs and throughputs should positively influence the outputs (or outcomes), which in this instance was an enriched training environment, and by extension, improved physiological and psychological measures.

\section{Aggregate and individual conditions}

Those in the aggregate condition trained in the presence of other participants; however, based on a rotation, these individuals consistently varied. This rotation was meant to minimize interaction between members, while providing a constant presence of co-exercisers. Those in the individual condition trained in isolation with a constant research assistant. As such, all study participants were involved in the same number of training hours, with the exception of the true group members taking part in the intervention session $(\sim 1 \mathrm{~h})$.

\section{Statistical analysis}

Data were analyzed using IBM SPSS for Windows (version 22; IBM Corp., Armonk, N.Y., USA). After testing for normality and variance homogeneity, descriptive statistics were computed. At the beginning of the study, 60 participants were involved in the program; however, because of various reasons (e.g., injury unrelated to training, other time commitments), 53 completed post-assessments. There were no significant differences $(p>0.05)$ at baseline between those that completed the training $(n=53)$ and those that did not $(n=7)$. With regard to the main analyses, and because of the baseline post-test design, analyses of covariance (ANCOVA) were computed, 
Table 1. Means and standard deviations (mean \pm SD) for anthropometric measures.

\begin{tabular}{llccc}
\hline & & \multicolumn{2}{l}{ Condition } & \\
\cline { 3 - 5 } Variable & Time & $\begin{array}{l}\text { True } \\
\text { group }\end{array}$ & Aggregate & $\begin{array}{l}\text { Training } \\
\text { alone }\end{array}$ \\
\hline Age $(\mathrm{y})$ & Pre & $21.9 \pm 3.6$ & $20.9 \pm 2.3$ & $23.1 \pm 2.5$ \\
Height $(\mathrm{cm})$ & Pre & $175.6 \pm 10.0$ & $173.7 \pm 9.3$ & $173.7 \pm 8.8$ \\
Body mass $(\mathrm{kg})$ & Pre & $74.4 \pm 14.0$ & $69.2 \pm 10.7$ & $75.1 \pm 10.0$ \\
& Post & $74.5 \pm 13.6$ & $69.3 \pm 10.5$ & $75.4 \pm 10.6$ \\
\hline
\end{tabular}

Note: Body mass did not change from pre-training (Pre) to posttraining (Post) in any group $(p>0.05)$.

Table 2. Means and standard deviations (mean \pm SD) for psychological variables.

\begin{tabular}{llccl}
\hline & \multicolumn{3}{l}{ Condition } \\
\cline { 3 - 5 } Variable & True & & Training \\
alone & group & Aggregate & alone \\
\hline Intrinsic motivation & Pre & $3.64 \pm 0.57$ & $3.25 \pm 0.69$ & $3.38 \pm 0.52$ \\
& Post & $3.74 \pm 0.44$ & $3.26 \pm 0.64$ & $3.18 \pm 0.71$ \\
Task self-efficacy & Pre & $94.18 \pm 7.47$ & $86.28 \pm 8.10$ & $87.09 \pm 12.31$ \\
& Post & $94.35 \pm 3.90$ & $96.17 \pm 3.71$ & $93.84 \pm 4.53$ \\
Scheduling self-efficacy & Pre & $92.24 \pm 9.83$ & $90.17 \pm 8.76$ & $83.69 \pm 13.37$ \\
& Post & $84.00 \pm 14.43$ & $92.20 \pm 6.84$ & $89.57 \pm 12.75$ \\
Groupness & Pre & $7.04 \pm 0.98$ & $5.40 \pm 1.43$ & - \\
& Post & $7.60 \pm 0.92$ & $6.80 \pm 0.90$ & - \\
\hline
\end{tabular}

Note: Intrinsic motivation is based on a 5-point scale (0-4), self-efficacy is based on a $100 \%$ response scale, and groupness is based on a 9-point scale (1-9). Pre, pre-training; Post, post-training.

with post-test scores as the dependent variable, groups as the independent variable, and baseline scores as the covariate (Dimitrov and Rumrill 2003). When no group interactions were found (i.e., the intervention did not elicit significant differences between groups), a time effect was still of interest to determine the effectiveness of SIT generally. As such, repeated-measures 1-way ANOVAs were computed to demonstrate improvements from baseline to post-test for all participants (Tabachnick and Fidell 2013).

\section{Results}

\section{Descriptive statistics}

Descriptive statistics (mean, standard deviation) for anthropometric and psychological variables for all conditions across time points are found in Tables 1 and 2, respectively. In addition, Figs. 1, 2 , and 3 depict the physiological measures for all groups at both time points. There was no difference in body mass between groups and no change with training $(p>0.05)$.

\section{Physiological measures}

After controlling for baseline levels, there was no significant main effect between groups for $\dot{V} O_{2 \max }\left(F_{[2,44]}=0.568, p>0.05, \eta^{2}=\right.$ 0.03). Despite this finding, it was of interest to determine the effectiveness of SIT within participants (Fig. 1), and as such, a repeated measure 1-way ANOVA identified a main effect for time $\left(F_{[1]}=15.834, p<0.001, \eta^{2}=0.03\right)$. Although there were no group differences, the individual condition improved their $\dot{\mathrm{V}} \mathrm{O}_{2 \max }$ by $2.5 \pm 4.4 \mathrm{~mL} \cdot \mathrm{kg}^{-1} \cdot \mathrm{min}^{-1}(95 \%$ confidence interval $(\mathrm{CI})=$ 0.27 to 4.76 ), the aggregate condition by $2.8 \pm 4.6 \mathrm{~mL} \cdot \mathrm{kg}^{-1} \cdot \mathrm{min}^{-1}$ (95\% CI $=0.73$ to 4.94 ), and the true group condition, by $2.5 \pm$ $4.4 \mathrm{~mL} \cdot \mathrm{kg}^{-1} \cdot \mathrm{min}^{-1}(95 \% \mathrm{CI}=0.48$ to 4.56$)$.

With regard to time-trial performance, after controlling for baseline levels, no statistically significant main effect was found $\left(F_{[2,47]}=2.432, p>0.05, \eta^{2}=0.09\right)$. Again, to determine time-trial performance across all participants (Fig. 2), a 1-way ANOVA identified a main effect for time $\left(F_{[1]}=28.611, p<0.001, \eta^{2}=0.37\right)$. Despite the lack of group differences, the individual condition
Fig. 1. Changes in maximal oxygen uptake $\left(\dot{V} \mathrm{O}_{2 \max }\right)\left(\mathrm{mL} \cdot \mathrm{kg}^{-1} \cdot \mathrm{min}^{-1}\right)$ from pre- to post-training. ${ }^{* *}$, Pre-post-training, $p<0.001$.

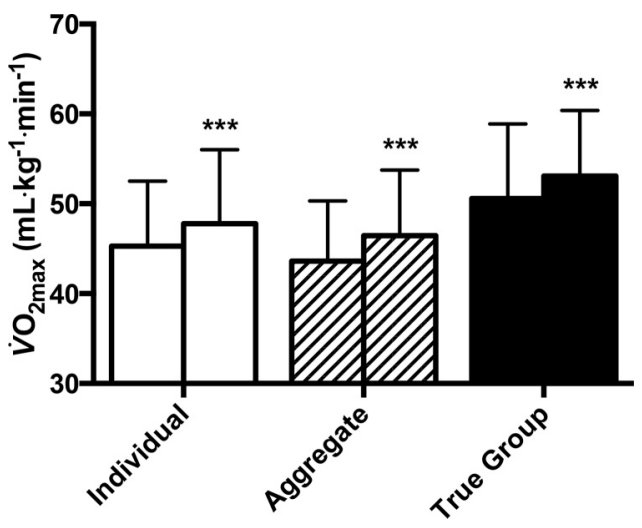

Fig. 2. Changes in 2000-m time-trial performance (s) from pre- to post-training. ${ }^{* *}$, Pre-post-training, $p<0.001$.

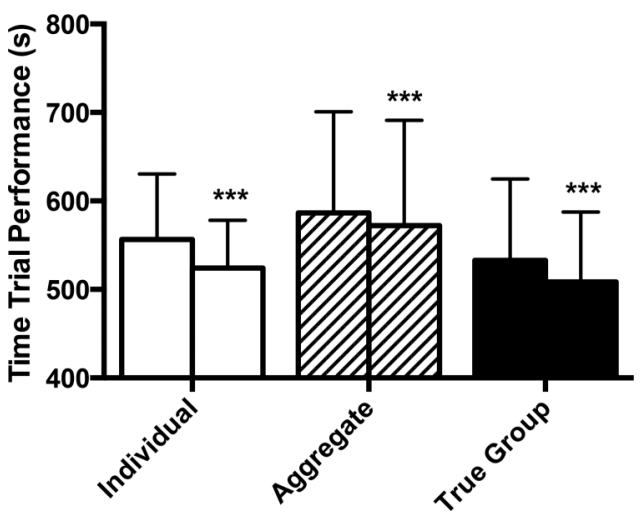

Fig. 3. Changes in peak speed during 30-s sprint test from pre- to post-training. $\mathrm{mph}$, miles per hour $(1 \mathrm{mile}=1.6 \mathrm{~km})$. ****, Pre-post-training, $p<0.001$.

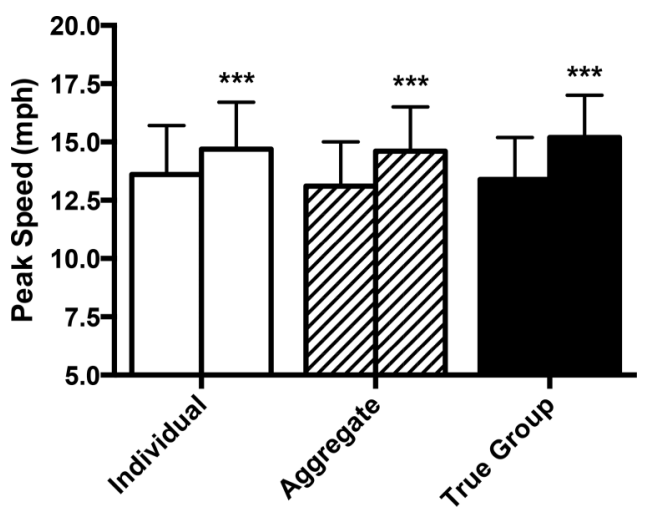

improved by $32.4 \pm 33.5 \mathrm{~s}(95 \% \mathrm{CI}=-49.3$ to -15.47$)$, the aggregate condition improved by $14.3 \pm 35.8 \mathrm{~s}(95 \% \mathrm{CI}=-30.85$ to 2.18$)$, and the true group condition improved by $24.2 \pm 21.0 \mathrm{~s}(95 \% \mathrm{CI}=-33.88$ to -14.45).

Finally, there was also no significant main effect for peak speed generated on the 30-s anaerobic sprint test $\left(F_{[2,47]}=1.255, p>0.05\right.$, $\left.\eta^{2}=0.05\right)$. However, all participants did significantly improve their peak speed output $\left(F_{[1]}=96.222, p<0.001, \eta^{2}=0.66\right.$; Fig. 3$)$, with the individual condition increasing $1.1 \pm 0.9 \mathrm{~km} \cdot \mathrm{h}^{-1}(95 \% \mathrm{CI}=0.7$ to 1.6$)$, the aggregate condition increasing $1.5 \pm 1.0 \mathrm{~km} \cdot \mathrm{h}^{-1}(95 \% \mathrm{CI}=1.1$ to 2.0$)$, and the true group condition increasing $1.7 \pm 1.2 \mathrm{~km} \cdot \mathrm{h}^{-1}(95 \% \mathrm{CI}=1.2$ to 2.3 ). 


\section{Psychological measures}

With regard to intrinsic motivation, results revealed a significant main effect between groups $\left(F_{[2,48]}=3.680, p=0.033, \eta^{2}=0.13\right)$. After controlling for baseline levels, the adjusted means indicated that improvements in the true group condition were significantly greater (residual change difference $=0.379, \mathrm{SE}=0.14, p=0.009,95 \% \mathrm{CI}=0.10$ to 0.66 ) than those in the individual condition. No other group differences were present $(p>.05)$.

For task self-efficacy, a significant main effect between groups was identified $\left(F_{[2,48]}=4.353, p=0.018, \eta^{2}=0.15\right)$, with the adjusted means demonstrating significantly greater improvements for the individual (residual change difference $=6.822, \mathrm{SE}=3.21, p=0.039$, $95 \% \mathrm{CI}=0.36$ to 13.28 ) and aggregate conditions (residual change difference $=8.654, \mathrm{SE}=3.11, p=0.008,95 \% \mathrm{CI}=2.39$ to 14.91$)$ in comparison with the true group condition. No significant group differences were found between the individual and aggregate conditions $(p>0.05)$.

A significant main effect between groups was also identified for scheduling self-efficacy $\left(F_{[2,48]}=6.630, p=0.003, \eta^{2}=0.22\right)$, whereby the individual (residual change difference $=11.017, \mathrm{SE}=3.42$, $p=0.002,95 \% \mathrm{CI}=4.13$ to 17.90 ) and aggregate conditions (residual change difference $=9.341, \mathrm{SE}=3.12, p=0.004,95 \% \mathrm{CI}=3.07$ to 15.61 ) were superior to the true group condition. Again, there was no significant difference between the individual and aggregate conditions $(p>0.05)$.

\section{Manipulation check}

After controlling for baseline levels, results indicated that there was no significant difference between the true group and aggregate conditions pertaining to perceptions of groupness $\left(F_{[1,34]}=\right.$ $0.343, p>0.05, \eta^{2}=0.01$ ). The aggregate group appears to have improved to a greater extent (residual change difference $=-0.232$, $\mathrm{SE}=0.32, p>0.05,95 \% \mathrm{CI}=-0.89$ to 0.42 ); however, the difference was not significant.

\section{Discussion}

The current project sought to assess the effectiveness of a group-enhanced SIT program for amateur athletes. More specifically, the purpose was to determine whether the development of true groups could influence both physiological and psychological outcomes to a greater extent than those obtained by individuals participating in aggregate and individual conditions. Generally, the group-based intervention failed to elicit differences between the conditions pertaining to physiological outcomes. Similarly, although differences did emerge for the psychological outcomes, they were not in the hypothesized direction.

Despite the unsuccessfulness of the group-based intervention, the SIT protocol itself was effective. Namely, in terms of $\dot{V O}_{2 \max }$, previous SIT studies have demonstrated improvements ranging from $4.2 \%$ to $13.4 \%$ (Gist et al. 2014; Sloth et al. 2013). In addition, a recent review identified effects of $\sim 6 \%$ from active nonathletic individuals (Weston et al. 2014b), which is quite similar to our training-induced improvements of 5.0\%-6.5\%. As such, our results are in-line with these as well as other running (Hazell et al. 2014a; MacPherson et al. 2011; Willoughby et al. 2016) and cycling (Astorino et al. 2011, 2012; Bailey et al. 2009; Burgomaster et al. 2005, 2008; Gibala et al. 2006; Hazell et al. 2010; Zelt et al. 2014) SIT studies lasting fewer than 6 weeks in duration. Similarly, the improvements found for time-trial performance $(2.4 \%-5.8 \%)$ were also of a similar magnitude to previous running (MacPherson et al. 2011; Willoughby et al. 2016) and cycling (Burgomaster et al. 2006) studies. Finally, peak running speed also increased with SIT training (8.3\%-12.8\%) consistently with previous work (Hazell et al. 2014a; MacPherson et al. 2011; Weston et al. 2014b; Willoughby et al. 2016). It is important to note that although actual training data were not measured because of logistical issues pertaining to the number of participants and space availability, previous studies have documented the training intensity achieved by this kind of SIT (Hazell et al. 2014b; Townsend et al. 2014,
Willoughby et al. 2016). Consequently, our results suggest the SIT protocol, regardless of experimental condition, resulted in improvements in-line with previous research.

Although physiological adaptations were demonstrated, the group-enhanced intervention failed to elicit improvements to a greater extent than those obtained within the aggregate and individual conditions. This was unexpected given the support for the influence of group processes on individual cognitions and behaviours in physical activity settings for a wide range of populations ranging from community to clinican based (Estabrooks et al. 2014). As previously discussed, physical activity interventions delivered to groups have shown to be more effective than when administered to individuals (Dishman and Buckworth 1996). In fact, a recent systematic review involving physical activity interventions that utilized group dynamics-based protocols found that of the 17 studies identified, 15 reported improvements in outcome variables (Estabrooks et al. 2011). Although studies referred to group dynamics strategies proposed by Carron and Spink (1993) - the framework followed within the current project - the authors indicated that a wide range of strategies were implemented across the studies, and used this to support the robust influence of this type of protocol (Estabrooks et al. 2011).

Whereas there is empirical support for the potential benefits derived from social processes, the current project sought to elicit changes in an amateur sport population - one dissimilar to those typically investigated in the exercise literature noted above. Interestingly, even though there is also support within the sport literature (Martin et al. 2009), this research has largely been conducted with intact teams. The current intervention integrated athletes from different teams and leagues interested in a new training modality, and attempted to develop new true groups from among them. Therefore, with the unusual combination of athletes involved in training without their teams and away from their typical environments, this study may have rendered the previous literature less relevant to this specific training intervention. Within the following sections, we advance several explanations as to why these unexpected results may have emerged.

With regard to the psychological variables, our hypotheses were not supported. Specifically, for motivation, although a visual inspection of the means does suggest a trend in the hypothesized direction - improvements in the true group, stability within the aggregates, and a decrease in the individuals - only the true group significantly differed from the individual conditions. However, the fact that the true group condition differed from the individual condition is significant considering the population of interest. These amateur athletes were motivated to train for their sports, and as such, had demonstrated high levels $(M=3.25-$ 3.64/4) of motivation at baseline, which did not provide sizeable opportunity for improvement. One explanation for this difference could be the group-based intervention in combination with the presence of others. This could explain why members in true groups were more motivated than the individuals training alone, but that there were no differences between the other conditions.

The nonsignificance found for motivation between the true group and the aggregate conditions was unexpected, but could perhaps be explained by social facilitation (Martin Ginis and Mack 2012) or social comparison (Osborn et al. 2012). As participants were constantly training in the presence of new exercisers, they could have felt a need to maintain self-presentation (Goffman 1959) throughout the program, which may have been less of an issue with members in the true group condition who became comfortable with one another. Another explanation could be that the team-building protocols did not facilitate a sense of unity among the members to a large enough extent to warrant changes in motivation over and above the presence of others. This is certainly evidenced by the nonsignificant manipulation check pertaining to groupness within these 2 conditions (i.e., true group vs. aggregate). 


\section{Pagination not final (cite DOI) / Pagination provisoire (citer le DOI)}

With regards to efficacy beliefs, these were interesting findings that could perhaps be attributed to the study protocol, and less to the effectiveness of the intervention. Specifically, based on the number of participants and because of space restrictions within the laboratory, those assigned to the true groups were provided with designated training times based on the availability of all group members. In contrast, those training alone or in the aggregate condition were afforded several times in which they could train. As scheduling self-efficacy reflects an individual's confidence in his/her ability to make time for and manage exercise behaviour (Rodgers et al. 2002), those in the true group condition may have felt more restricted as the training program progressed.

Another issue pertaining to the intervention could have been the assignment of athletes to the true group condition. As previously indicated, participants were assigned to 1 of 3 conditions based on $\dot{V} \mathrm{O}_{2 \max }$, sex, and time-trial performance. As such, no consideration was made for group processes such as individual similarity (Dunlop and Beauchamp 2013), which may have been a limitation given that recent work with athletes suggests the importance of similar characteristics, personalities, and previous experiences for grouping tendencies (Martin et al. 2015). The aggregate and individual conditions would likely not have been affected by this assignment, as they had little (or no) interaction with other athletes. Within the true group condition, however, a more thoughtful approach to assigning athletes based on similar sports, leagues, or previous experiences (i.e., established relations) could have improved the training environment. Although there are certainly benefits to increased diversity among group members, such as increased amounts and variability of resources (Forsyth 2014), for relatively basic tasks such as sprint training, the most important element for the group's composition may be compatibility (Widmeyer and Loy 1989).

A final issue worth discussing involves the assessment of groupness. Notably, the difference between conditions was nonsignificant. We hypothesized that in order for the social processes to enrich the training environment, those in the true groups would need to demonstrate that they perceived themselves as being a group to a greater extent that the aggregate condition, but this was not the case. This was unexpected, as the group-based intervention was designed to create a sense of unity among the true group members, and to avoid quality interaction and group-oriented perceptions among the aggregate condition. Interestingly, the mean for the aggregate condition indicates a high level of groupness for individuals who did not have prolonged or quality exposure to one another $(M=6.80 / 9)$. Perhaps the provision of a groupness questionnaire at baseline and the subsequent presence of co-exercisers during the training sessions inadvertently led to favourable responses based on social desirability at post-testing. Similarly, although there was rotation in the aggregate condition, the facility is small, meaning that proximity could have increased interaction among those training at the same time.

As a summary, although unexpected results emerged pertaining to the effectiveness of the group-based intervention, the SIT protocol did elicit physiological improvements for those involved. This study recognized the interaction between physiological and psychological outcomes, and represents a novel attempt at manipulating the training environment for a modality that is growing in popularity. In terms of training programs, the current sample was moderately large and was composed of equal numbers of males and females. Assignment to training condition was based on physiological measures, and perhaps future research should consider other forms of grouping criteria (e.g., participant similarity, established relationships).

\section{Perspective}

SIT is a training modality that has received an increased amount of research attention in physiology (Gibala et al. 2014), and more recently in psychology (Biddle and Batterham 2015; Hardcastle et al. 2014). There is relative consensus pertaining to the training adapta- tions derived from SIT (Gibala et al. 2014), and although questions pertaining to affective responses are beginning to receive answers (Martinez et al. 2015), others pertaining to psychological implications remain (Del Vecchio et al. 2015; Hardcastle et al. 2014; Jung et al. 2015). This project represents the first (of our knowledge) to attempt to enrich the training environment through social processes for an athlete population. Despite the lack of influence pertaining to the group-based intervention, researchers are provided with a template from which to improve upon for future study. This is a relevant line of inquiry within sport psychology, as a group of experts has recently indicated that group dynamics-related topics are underrepresented within the field (Kleinert et al. 2012).

\section{Conflict of interest statement}

The authors declare that there are no conflicts of interest.

\section{Acknowledgements}

We would like to thank the Sport Science Association of Alberta (SSAA) for the funding of this project, and Katie Couture for her assistance with data collection.

\section{References}

Astorino, T.A., Allen, R.P., Roberson, D.W., Jurancich, M., Lewis, R., McCarthy, K., and Trost, E. 2011. Adaptations to high-intensity training are independent of gender. Eur. J. Appl. Physiol. 111(7): 1279-1286. doi:10.1007/s00421-010-1741-y. PMID:21132441.

Astorino, T.A., Allen, R.P., Roberson, D.W., and Jurancich, M. 2012. Effect of high-intensity interval training on cardiovascular function, VO2max, and muscular force. J. Strength Cond. Res. 26(1): 138-145. doi:10.1519/JSC. 0b013e318218dd77. PMID:22201691.

Bailey, S.J., Wilkerson, D.P., DiMenna, F.J., and Jones, A.M. 2009. Influence of repeated sprint training on pulmonary $\mathrm{O}_{2}$ uptake and muscle deoxygenation kinetics in humans. J. Appl. Physiol. (1985), 106(6): 1875-1887. doi:10.1152/ japplphysiol.00144.2009. PMID:19342439.

Biddle, S.J., and Batterham, A.M. 2015. High-intensity interval exercise training for public health: a big HIT or shall we HIT it on the head? Int. J. Behav. Nutr. Phys. Act. 12(1): 95. doi:10.1186/s12966-015-0254-9. PMID:26187579.

Burgomaster, K.A., Hughes, S.C., Heigenhauser, G.J., Bradwell, S.N., and Gibala, M.J. 2005. Six sessions of sprint interval training increases muscle oxidative potential and cycle endurance capacity in humans. J. Appl. Physiol. 98(6): 1985-1990. doi:10.1152/japplphysiol.01095.2004. PMID:15705728.

Burgomaster, K.A., Heigenhauser, G.J., and Gibala, M.J. 2006. Effect of short-term sprint interval training on human skeletal muscle carbohydrate metabolism during exercise and time-trial performance. J. Appl. Physiol. 100(6): 20412047. doi:10.1152/japplphysiol.01220.2005. PMID:16469933.

Burgomaster, K.A., Howarth, K.R., Phillips, S.M., Rakobowchuk, M., MacDonald, M.J., McGee, S.L., and Gibala, M.J. 2008. Similar metabolic adaptations during exercise after low volume sprint interval and traditional endurance training in humans. J. Physiol. 586(1): 151-160. doi:10.1113/jphysiol.2007. 142109. PMID:17991697.

Burke, S.M., Carron, A.V., Eys, M.A., Ntoumanis, N., and Estabrooks, P.A. 2006. Group versus individual approach? A meta-analysis of the effectiveness of interventions to promote physical activity. Sport Exerc. Psychol. Rev. 2(1): 19-35.

Campbell, D.T. 1958. Common fate, similarity, and other indexes of the status of aggregates of persons as social entities. Behav. Sci. 3(1): 14-25.

Carron, A.V., and Eys, M.A. 2012. Group Dynamics in Sport. 4th ed. Fitness Information Technology, Morgantown, W. Va., USA.

Carron, A.V., and Hausenblas, H.A. 1998. Group Dynamics in Sport. 2nd ed. Fitness Information Technology, Morgantown, W. Va., USA.

Carron, A.V., and Spink, K.S. 1993. Team building in an exercise setting. Sport Psychol. 7(1): 8-18.

Crozier, A.J., Spink, K.S., Wilson, K.S., Ulvick, J.D., and Priebe, C.S. 2012. “All for one": Examining the effects of cohesion and groupness on adherence in structured exercise settings. J. Sport Exerc. Psychol. 34: S219-S219.

Del Vecchio, F.B., Gentil, P., Coswig, V.S., and Fukuda, D.H. 2015. Commentary: Why sprint interval training is inappropriate for a largely sedentary population. Front. Psychol. 6: 1359. PMID:26441735.

Dimitrov, D.M., and Rumrill, P.D., Jr. 2003. Pretest-posttest designs and measurement of change. Work, 20(2): 159-165. PMID:12671209.

Dishman, R.K., and Buckworth, J. 1996. Increasing physical activity: a quantitative synthesis. Med. Sci. Sports Exerc. 28(6): 706-719. doi:10.1097/00005768199606000-00010. PMID:8784759.

Dunlop, W.L., and Beauchamp, M.R. 2013. Birds of a feather stay active together: a case study of an all-male older adult exercise program. J. Aging Phys. Act. 21(2): 222-232. PMID:22899819.

Estabrooks, P.A., and Glasgow, R.E. 2006. Translating effective clinic-based phys- 
ical activity interventions into practice. Am. J. Prev. Med. 31(4): S45-S56. PMID:16979469.

Estabrooks, P.A., and Smith-Ray, R.L. 2005. Clinical social cognitive physical activity interventions. Ann. Behav. Med. 29: SO30.

Estabrooks, P.A., Almeida, F., Schriener, P., Gonzales, M., Van Den Berg, R., Onda, P. and Smith-Ray, R. 2006. Designing for dissemination: the process of translating a clinic-based physical activity intervention into practice. Med. Sci. Sports Exerc. 38(5): S367-S367. doi:10.1249/00005768-200605001-01558.

Estabrooks, P.A., Bradshaw, M., Dzewaltowski, D.A., and Smith-Ray, R.L. 2008. Determining the impact of Walk Kansas: applying a team-building approach to community physical activity promotion. Ann. Behav. Med. 36(1): 1-12. doi:10.1007/s12160-008-9040-0. PMID:18607666.

Estabrooks, P.A., Smith-Ray, R.L., Almeida, F.A., Hill, J., Gonzales, M., Schreiner, P., and Van Den Berg, R. 2011. Move more: translating an efficacious group dynamics physical activity intervention into effective clinical practice. Int. J. Sport Exerc. Pysch. 9: 4-18. doi:10.1080/1612197X.2011.563123.

Estabrooks, P.A., Harden, S.M., Johnson, S.B., and Pardo, K.A. 2014. Group integration interventions in Exercise: Theory, practice, and future directions. In Group Dynamics in Exercise and Sport Psychology. 2nd ed. Edited by M.R. Beauchamp and M.A. Eys. Routledge, New York, N.Y., USA. pp. 164-182.

Forsyth, D.R. 2014. Group Dynamics. 6th ed. Cengage Learning, Wadsworth, Calif., USA.

Forsyth, D.R., and Burnett, J.L. 2010. Group processes. In Advanced Social Psychology: The State of the Science. Edited by E.R. Baumeister and E. Finkel. Oxford University Press, New York, N.Y., USA. pp. 495-534.

Gibala, M.J., Little, J.P., van Essen, M., Wilkin, G.P., Burgomaster, K.A., Safdar, A., et al. 2006. Short-term sprint interval versus traditional endurance training: similar initial adaptations in human skeletal muscle and exercise performance. J. Physiol. 575(3): 901-911. doi:10.1113/jphysiol.2006.112094. PMID: 16825308 .

Gibala, M.J., Gillen, J.B., and Percival, M.E. 2014. Physiological and health-related adaptations to low-volume interval training: influences of nutrition and sex. Sports Med. 44(S2): S127-S137. PMID:25355187.

Gillen, J.B., Percival, M.E., Skelly, L.E., Martin, B.J., Tan, R.B., Tarnopolsky, M.A., and Gibala, M.J. 2014. Three minutes of all-out intermittent exercise per week increases skeletal muscle oxidative capacity and improves cardiometabolic health. PLoS ONE, 9(11): e111489. doi:10.1371/journal.pone.0111489. PMID: 25365337.

Gist, N.H., Fedewa, M.V., Dishman, R.K., and Cureton, K.J. 2014. Sprint interval training effects on aerobic capacity: a systematic review and meta-analysis. Sports Med. 44(2): 269-279. doi:10.1007/s40279-013-0115-0. PMID:24129784.

Goffman, E. 1959. The Presentation of Self in Everyday Life. Doubleday, Garden City, N.Y., USA.

Hardcastle, S.J., Ray, H., Beale, L., and Hagger, M.S. 2014. Why sprint interval training is inappropriate for a largely sedentary population. Front. Psychol. 5: 1505. PMID:25566166.

Harris, R.C., Edwards, R.H., Hultman, E., Nordesjo, L.O., Nylind, B., and Sahlin, K. 1976. The time course of phosphorylcreatine resynthesis during recovery of the quadriceps muscle in man. Pflugers Arch. 367(2): 137-142. doi:10.1007| BF00585149. PMID:1034909.

Hazell, T.J., MacPherson, R.E., Gravelle, B.M., and Lemon, P.W. 2010. 10 or 30-s sprint interval training bouts enhance both aerobic and anaerobic performance. Eur. J. Appl. Physiol. 110(1): 153-160. doi:10.1007/s00421-010-1474-y. PMID:20424855.

Hazell, T.J., Hamilton, C.D., Olver, T.D., and Lemon, P.W. 2014a. Running sprint interval training induces fat loss in women. Appl. Physiol. Nutr. Metab. 39(8): 944-950. doi:10.1139/apnm-2013-0503. PMID:24905559.

Hazell, T.J., Olver, T.D., Macpherson, R.E., Hamilton, C.D., and Lemon, P.W. 2014b. Sprint interval exercise elicits near maximal peak VO2 during repeated bouts with a rapid recovery within 2 minutes. J. Sports Med. Phys. Fitness, 54(6): 750-756. PMID:25350032.

Jung, M.E., Little, J.P., and Batterham, A.M. 2015. Commentary: Why sprint interval training is inappropriate for a largely sedentary population. Front. Psychol. 6: 1999. PMID:26779101.

Kleinert, J., Ohlert, J., Carron, A.V., Eys, M.A., Feltz, D.L., Hardwood, C., et al. 2012. Group dynamics in sport: an overview and recommendations on diagnostic and intervention. Sport Psychol. 26: 412-434.

Lox, C.L., Martin Ginis, K.A., and Petruzzello, S.J. 2014. The Psychology of Exercise: Integrating Theory and Practice. 4 th ed. Holcomb Hathaway, Scottsdale, Ariz., USA.

MacPherson, R.E., Hazell, T.J., Olver, T.D., Paterson, D.H., and Lemon, P.W. 2011. Run sprint interval training improves aerobic performance but not maximal cardiac output. Med. Sci. Sports Exerc. 43(1): 115-122. PMID:20473222.

Marcos, F.M., Miguel, P.A., Oliva, D.S., and Calvo, T.G. 2010. Interactive effects of team cohesion on perceived efficacy in semi-professional sport. J. Sports Sci. Med. 9(2): 320-325. PMID:24149702.

Markland, D., and Tobin, V. 2004. A modification to the behavioural regulation in exercise questionnaire to include an assessment of amotivation. J. Sport Exerc. Psychol. 26(2): 191-196.

Martin, L.J., Carron, A.V., and Burke, S.M. 2009. Team building interventions in sport: a meta-analysis. Sport Exerc. Psychol. Rev. 5: 3-18.

Martin, L.J., Wilson, J., Evans, M.B., and Spink, K.S. 2015. Cliques in sport: Perceptions of intercollegiate athletes. Sport Psychol. 29: 82-95. doi:10.1123/tsp. 2014-0003.

Martin Ginis, K.A., and Mack, D. 2012. Understanding exercise behavior: A selfpresentational perspective. In Advances in Motivation in Sport and Exercise. Edited by G.C. Roberts and D.C. Treasure. Human Kinetics, Champaign Ill., USA. pp. 327-355.

Martinez, N., Kilpatrick, M.W., Salomon, K., Jung, M.E., and Little, J.P. 2015. Affective and enjoyment responses to high-intensity interval training in overweight-to-obese and insufficiently active adults. J. Sport Exerc. Psychol. 37(2): 138-149. doi:10.1123/jsep.2014-0212. PMID:25996105.

Midgley, A.W., McNaughton, L.R., Polman, R., and Marchant, D. 2007. Criteria for determination of maximal oxygen uptake: a brief critique and recommendations for future research. Sports Med. 37(12): 1019-1028. doi:10.2165/00007256200737120-00002. PMID:18027991.

Osborn, K.A., Irwin, B.C., Skogsberg, N.J., and Feltz, D.L. 2012. The Köhler effect: motivation gains and losses in real sports groups. Sport Exerc. Perf. Rev. 1(4): 242-253.

Prapavessis, H., and Carron, A.V. 1997. Cohesion and work output. Small Group Res. 28(2): 294-301. doi:10.1177/1046496497282006.

Priebe, C.S., Spink, K.S., and DeRoo, T. 2011. An exploratory study examining groupness, cohesion, and satisfaction in an exercise setting. J. Sport Exerc. Psychol. 33: S179-S180.

Rhea, M.R., Landers, D.M., Alvar, B.A., and Arent, S.M. 2003. The effects of competition and the presence of an audience on weight lifting performance. J. Strength Cond Res. 17(2): 303-306. PMID:12741867.

Rodgers, W.M., and Sullivan, M.J. 2001. Task, coping, and scheduling self-efficacy in relation to frequency of physical activity. J. Appl. Soc. Psych. 31: 741-753. doi:10.1111/j.1559-1816.2001.tb01411.x.

Rodgers, W.M., Hall, C.R., Blanchard, C.M., McAuley, E., and Munroe, K.J. 2002. Task and scheduling self-efficacy as predictors of exercise behaviour. Pysch. Health, 17(4): 405-416. doi:10.1080/0887044022000004902.

Sloth, M., Sloth, D., Overgaard, K., and Dalgas, U. 2013. Effects of sprint interval training on $\mathrm{VO}_{2 \max }$ and aerobic exercise performance: a systematic review and meta-analysis. Scand. J. Med. Sci. Sports, 23(6): e341-e352. doi:10.1111/sms. 12092.

Spink, K.S. 1995. Cohesion and intention to participate of female sport athletes. J. Sport Exerc. Psychol. 31(4): 741-753.

Spink, K.S., Wilson, K.S., and Priebe, C.S. 2010. Groupness and adherence in structured exercise settings. Group Dyn. Theory Res. Pract. 14(2): 163-173. doi:10.1037/a0017596.

Tabachnick, B.G., and Fidell, L.S. 2013. Using Multivariate Statistics. 6th ed. Pearson, N.J., USA.

Townsend, L.K., Couture, K.M., and Hazell, T.J. 2014. Mode of exercise and sex are not important for oxygen consumption during and in recovery from sprint interval training. Appl. Physiol. Nutr. Metab. 39(12): 1388-1394. doi:10.1139/ apnm-2014-0145. PMID:25386979.

Viglietta, R.C., Wilson, K.S., Spink, K.S., Ulvick, J.D., Crozier, A.J., and Priebe, C.S. 2012. Would I come back? The role of groupness and cohesion in intention to return. J. Sport Exerc. Psychol. 34: S297-S298.

Weston, K.S., Wisloff, U., and Coombes, J.S. 2014a. High-intensity interval training in patients with lifestyle-induced cardiometabolic disease: a systematic review and meta-analysis. Br. J. Sports Med. 48(16): 1227-1234. doi:10.1136/ bjsports-2013-092576. PMID:24144531.

Weston, M., Taylor, K.L., Batterham, A.M., and Hopkins, W.G. 2014b. Effects of low-volume high-intensity interval training (HIT) on fitness in adults: a metaanalysis of controlled and non-controlled trials. Sports Med. 44(7): 1005-1017. doi:10.1007/s40279-014-0180-z. PMID:24743927.

Widmeyer, W.N., and Loy, J.W. 1989. Dynamic duos: An analysis of the relationship between group composition and group performance in women's doubles tennis. In Studies in Honor of J. M. Roberts. Edited by R. Bolton. Human Relations Area Files, New Haven, Conn., USA.

Willoughby, T.N., Thomas, M.P.L., Schmale, M.S., Copeland, J.L., and Hazell, T.J. 2016. Four weeks of running sprint interval training improves cardiorespiratory fitness in young and middle-aged adults. J. Sport Sci. 34: 1207-1214. doi:10.1080/02640414.2015.1102316.

Zelt, J.G., Hankinson, P.B., Foster, W.S., Williams, C.B., Reynolds, J., Garneys, E., et al. 2014. Reducing the volume of sprint interval training does not diminish maximal and submaximal performance gains in healthy men. Eur. J. Appl. Physiol. 114(11): 2427-2436. doi:10.1007/s00421-014-2960-4. PMID:25091854. 